PROCEEDINGS OF THE

AMERICAN MATHEMATICAL SOCIETY

Volume 125, Number 10, October 1997, Pages 2839-2841

S 0002-9939(97)04023-9

\title{
EXACT TOPOLOGICAL ANALOGS TO ORTHOPOSETS
}

\author{
PETER G. OVCHINNIKOV
}

(Communicated by Franklin D. Tall)

\begin{abstract}
An arbitrary orthoposet $E$ is shown to be isomorphic to $\left(\mathcal{E}, \subset{ }^{c}{ }^{c}\right)$, $\mathcal{E}$ being a subbasis of a Hausdorff topological space $\mathcal{S}$ satisfying 1) $\mathcal{S} \in \mathcal{E}, 2$ ) $\alpha \in \mathcal{E} \Rightarrow \alpha^{c} \in \mathcal{E}$, and 3) every covering of $\mathcal{S}$ by elements of $\mathcal{E}$ possesses an at most 2 -element subcovering. The couple $(\mathcal{S}, \mathcal{E})$ turns out to be unique.
\end{abstract}

\section{INTRODUCTION}

A well-known theorem by M. Stone (cf. $[2,8]$ ) establishes a bijective correspondence between the Boolean algebras and the zero-dimensional compact topological spaces. Thus these spaces serve to be exact topological analogs to the Boolean algebras. Let us widen the class of the Boolean algebras to the class of all orthoposets (cf. $[2,4,5])$. Which will be an exact topological "double" to the latter class?

An important class of orthoposets wider than the Boolean algebras is the class of orthomodular lattices (cf. [4]). It was proved in [1] that for every orthomodular lattice $L$ there exists a compact Hausdorff closure (not necessarily topological) space $\mathcal{L}$ such that the orthomodular lattice $C O(\mathcal{L})$ of all clopen subsets of $\mathcal{L}$ is isomorphic to $L$.

There are many orthoposets which are not Boolean algebras or even orthomodular lattices (cf. $[3,4,5,7]$ ). As is well-known, they are of importance within the foundations of quantum mechanics (cf. [3, 7]).

In Section 2 of this note, we introduce a purely topological concept of an orthopair which enables one to consider a bijective correspondence between the orthoposets and the orthopairs.

\section{Orthopairs}

Recall that an orthoposet (cf. $[2,4,5]$ ) is a poset $E$ with the greatest element 1 and an involutive antiautomorphism ' $: E \rightarrow E$ satisfying $x \vee x^{\prime}=1$ for every $x \in E$.

Also recall that two orthoposets, $E_{1}$ and $E_{2}$, are said to be isomorphic if there exists an isomorphism between them, i.e., a bijection $\varphi: E_{1} \rightarrow E_{2}$ satisfying $x \leq y \Leftrightarrow \varphi(x) \leq \varphi(y)\left(x, y \in E_{1}\right)$ and $\varphi\left(x^{\prime}\right)=\varphi(x)^{\prime}\left(x \in E_{1}\right)$.

Received by the editors April 9, 1996.

1991 Mathematics Subject Classification. Primary 06C15, 54H10; Secondary 81P10.

Key words and phrases. Orthopair, orthoposet, subbasis, zero-dimensional compact topological space. 
Definition 2.1. An orthopair is a couple $(\mathcal{S}, \mathcal{E})$, wherein $\mathcal{S}$ is a Hausdorff topological space and $\mathcal{E}$ is a subbasis of $\mathcal{S}$ satisfying

1) $\mathcal{S} \in \mathcal{E}$

2) $\alpha \in \mathcal{E} \Rightarrow \alpha^{c} \in \mathcal{E}$ (here, $\alpha^{c}=\mathcal{S} \backslash \alpha$ ), and

3 ) every covering of $\mathcal{S}$ by elements of $\mathcal{E}$ possesses an at most 2-element subcovering.

Remark 2.2. Suppose that $(\mathcal{S}, \mathcal{E})$ is an orthopair. Then $\left(\mathcal{E}, \subset{ }^{c}\right)$ is obviously an orthoposet and $\mathcal{S}$ is zero-dimensional (i.e., has a basis consisting of clopen sets). By the Alexander Lemma (cf. $[2,6]), \mathcal{S}$ is compact.

Theorem 2.3. For an arbitrary orthoposet $E$ there exists an orthopair $(\mathcal{S}, \mathcal{E})$ such that $E$ and $\mathcal{E}$, as orthoposets, are isomorphic. Moreover, if $\left(S_{1}, \mathcal{E}_{1}\right)$ and $\left(\mathcal{S}_{2}, \mathcal{E}_{2}\right)$ are orthopairs such that $\mathcal{E}_{1}$ and $\mathcal{E}_{2}$ are both isomorphic to $E$, then there exists a homeomorphism $u: \mathcal{S}_{1} \rightarrow \mathcal{S}_{2}$ satisfying $\alpha \in \mathcal{E}_{1} \Leftrightarrow u(\alpha) \in \mathcal{E}_{2}$ for every $\alpha \subset \mathcal{S}_{1}$.

Proof. Let $E$ be an orthoposet. Put $\mathcal{S}=\{f: E \rightarrow\{0,1\} \mid f$ is monotonic and $f(x)+f\left(x^{\prime}\right)=1$ for all $\left.x \in E\right\}$. Note that $f \in \mathcal{S} \Rightarrow f(1)=1$. We consider $\mathcal{S}$ as a subspace of the product topological space $\{0,1\}^{E}$. Obviously $\mathcal{S}$ is Hausdorff. For every $x \in E$, we define a continuous mapping $\pi_{x}: \mathcal{S} \rightarrow\{0,1\}$ as $\pi_{x}(f)=f(x)$ $(f \in \mathcal{S})$. Define a mapping $\varphi: E \rightarrow \mathcal{P}(\mathcal{S}), \mathcal{P}(\mathcal{S})$ being the set of all subsets of $\mathcal{S}$ by $\varphi(x)=\pi_{x}^{-1}(1)(x \in E)$. Put $\mathcal{E}=\{\varphi(x) \mid x \in E\}$. Then $\mathcal{E}$ is obviously a subbasis of $\mathcal{S}$. Next, $\mathcal{S}=\varphi(1) \in \mathcal{E}$. Since $\left.\varphi(x)^{c}=\varphi\left(x^{\prime}\right)(x \in E), 2\right)$ follows. Obviously $x, y \in E, x \leq y \Rightarrow \varphi(x) \subset \varphi(y)$.

We aim to show that $x, y \in E, \varphi(x) \subset \varphi(y) \Rightarrow x \leq y$ and to establish 3). Prior to this, we need a lemma.

For $x, y \in E$, we write $x \perp y$ provided that $x \leq y^{\prime}$ and $x \not \perp y$ otherwise.

Lemma 2.4. Let $A \subset E$ satisfy $x, y \in A \Rightarrow x \not \perp y$. Then there exists $f \in \mathcal{S}$ with $A \subset f^{-1}(1)$.

Proof. By the Zorn Lemma, there is a maximal, with respect to inclusion, $B \subset E$ such that $A \subset B$ and $x, y \in B \Rightarrow x \not \perp y$. Put

$$
f(x)= \begin{cases}1 & \text { if } x \in B \\ 0 & \text { if } x \in E \backslash B .\end{cases}
$$

It suffices to show that

(i) $x \in B, y \in E, x \leq y \Rightarrow y \in B$ and

(ii) if $x \in E$, then either $x \in B$ or $x^{\prime} \in B$.

Suppose that (ii) fails to be true. Then there obviously exists $x \in E$ with $x \notin B$ and $x^{\prime} \notin B$. Since $B$ is maximal, there exist $z, a \in B$ with $z \perp x$ and $x^{\prime} \perp a$. Then $z \perp a$. This is a contradiction. Thus (ii) follows. Let us prove (i). Suppose that $x \in B, y \in E$, and $x \leq y$. Since $x \perp y^{\prime}$, we get $y^{\prime} \notin B$. By (ii), $y \in B$.

Let us continue the proof of the theorem. Let $x, y \in E$ satisfy $\varphi(x) \subset \varphi(y)$. Suppose $x \not \leq y$. Since $x \not \not y^{\prime}$, by Lemma 2.4, there exists $f \in \mathcal{S}$ satisfying $f(x)=$ $f\left(y^{\prime}\right)=1$. Obviously $f \in \varphi(x) \backslash \varphi(y)$. This is a contradiction. Since we have verified 1) and 2) and have shown $\varphi$ to satisfy $x \leq y \Leftrightarrow \varphi(x) \subset \varphi(y)(x, y \in E)$ and $\varphi\left(x^{\prime}\right)=\varphi(x)^{c}(x \in E)$, it follows that $\left(\mathcal{E}, \subset,{ }^{c}\right)$ is an orthoposet and $\varphi$ is an isomorphism between $E$ and $\mathcal{E}$. Let us show 3). Suppose that $\mathcal{A} \subset \mathcal{E}$ is a covering of $\mathcal{S}$ which contains no at most 2-element subcovering. Put $\mathcal{B}=\left\{\alpha^{c} \mid \alpha \in \mathcal{A}\right\}$. Since $\beta \cap \gamma \neq \emptyset(\beta, \gamma \in \mathcal{B})$, it follows that $\varphi^{-1}(\beta) \not \perp \varphi^{-1}(\gamma)(\beta, \gamma \in \mathcal{B})$. By 
Lemma 2.4, there exists $f \in \mathcal{S}$ with $f\left(\varphi^{-1}(\beta)\right)=1$ for all $\beta \in \mathcal{B}$. Thus we obtain $f \in \bigcap_{\beta \in \mathcal{B}} \beta=\left(\bigcup_{\alpha \in \mathcal{A}} \alpha\right)^{c}$. This is a contradiction.

Now, take an arbitrary orthopair $(T, \mathcal{F})$. Put $\mathcal{S}_{1}=\{f: \mathcal{F} \rightarrow\{0,1\} \mid \alpha, \beta \in$ $\mathcal{F}, \alpha \subset \beta \Rightarrow f(\alpha) \leq f(\beta)$ and $\left.\alpha \in \mathcal{F} \Rightarrow f(\alpha)+f\left(\alpha^{c}\right)=1\right\}$. We endow $\mathcal{S}_{1}$ with the topology inherited from $\{0,1\}^{\mathcal{F}}$. By the Tikhonoff Theorem, $\mathcal{S}_{1}$ is compact. For each $t \in T$ define $f_{t} \in \mathcal{S}_{1}$ by

$$
f_{t}(\alpha)=\left\{\begin{array}{ll}
1 & \text { if } t \in \alpha, \\
0 & \text { if } t \notin \alpha
\end{array} \quad(\alpha \in \mathcal{F}) .\right.
$$

Since the sets $\alpha \in \mathcal{F}$ are clopen and $T$ is Hausdorff, it follows that the mapping $\phi: T \rightarrow \mathcal{S}_{1}$ defined as $\phi(t)=f_{t}(t \in T)$ is a continuous injection. Suppose $f \in \mathcal{S}_{1}$. Put $\mathcal{A}_{f}=\{\alpha \in \mathcal{F} \mid f(\alpha)=0\}$. If $\alpha, \beta \in \mathcal{A}_{f}$, then $f\left(\alpha^{c}\right)=1>f(\beta)$ and thus $\alpha^{c} \not \subset \beta$, i.e., $\alpha \cup \beta \neq T$. Hence $\mathcal{A}_{f}$ is not a covering of $T$. If we take $t \in T \backslash\left(\bigcup_{\alpha \in \mathcal{A}_{f}} \alpha\right)$, then $f_{t}=f$. Thus $\phi$ is a bijection. As $T$ and $\mathcal{S}_{1}$ are compact, $\phi$ is a homeomorphism.

To conclude the proof of the theorem, suppose that $\psi: \mathcal{F} \rightarrow E$ is an isomorphism. Put $u(f)=\phi^{-1}(f \circ \psi)(f \in \mathcal{S})$. Obviously $u: \mathcal{S} \rightarrow T$ is a homeomorphism, and if $\alpha \subset \mathcal{S}$, then $\alpha \in \mathcal{E} \Leftrightarrow u(\alpha) \in \mathcal{F}$.

Remark 2.5. By Lemma 2.4, we may identify the functions $f \in \mathcal{S}$ with the maximal, with respect to inclusion, subsets $A \subset E$ with $x, y \in A \Rightarrow x \not \perp y$.

Example 2.6. Let $E$ be the Boolean algebra of all subsets of $\{1,2,3\}$. Observe that $(\{1,2,3\}, E),\{1,2,3\}$ being endowed with the discrete topology is not an orthopair, though $\{1,2,3\}$ is a Stone space for $E$. By making use of Remark 2.5, let us construct an orthopair, $(\mathcal{S}, \mathcal{E})$, for $E$. Put $A=\{\{1\},\{1,2\},\{1,3\},\{1,2,3\}\}$, $B=\{\{2\},\{1,2\},\{2,3\},\{1,2,3\}\}, C=\{\{3\},\{1,3\},\{2,3\},\{1,2,3\}\}$, and $D=$ $\{\{1,2\},\{2,3\},\{1,3\},\{1,2,3\}\}$. Then $\mathcal{S}=\{A, B, C, D\}$, the topology in $\mathcal{S}$ is discrete, and $\mathcal{E}=\{\emptyset,\{A\},\{B\},\{C\},\{A, B, D\},\{B, C, D\},\{A, C, D\}, \mathcal{S}\}$.

\section{ACKNOWLEDGMENT}

The author gratefully acknowledges the support of the grant no. 95-01-00025 of the Russian Foundation for Fundamental Research.

\section{REFERENCES}

[1] J. Binder and P. Pták, A representation of orthomodular lattices, Acta Univ. Carolinae 31 (1990), 21-26. MR 92b:06028

[2] G. Birkhoff, Lattice theory, AMS, Providence, RI, 1967. MR 37:2638

[3] S. Gudder, Stochastic methods in quantum mechanics, North Holland, New York, 1979. MR 84j:81003

[4] G. Kalmbach, Orthomodular lattices, Academic Press, London, 1983. MR 85f:06012

[5] _ Measures and Hilbert lattices, World Scientific, Singapore, 1986. MR 88a:06013

[6] K. Kuratowski, Topology, v. 2, Academic Press, New York, 1968. MR 41:4467

[7] P. Pták and S. Pulmannová, Orthomodular structures as quantum logics, Kluwer, Dordrecht, 1991. MR 94d:81018b

[8] R. Sikorski, Boolean algebras, Springer, Berlin, 1964. MR 31:2178

Department of Mathematics, Kazan State University, 420008, Kazan, Russia

E-mail address: Petr.Ovchinnikov@ksu.ru 